J-MACS (Japanese registry for mechanically assisted circulatory support) has listed a total of 722 patients between December 2010 and October 2017. Mean age was 43.2 years and mean body surface area was $1.63 \mathrm{~m}^{2}$. Aetiology of heart failure was dilated cardiomyopathy in $77 \%$ and ischaemic cardiomyopathy in 11\%. Pre-operative INTERMACS profile was level 1 in 10\%, level 2 in 39\%, level 3 in 48\%, and level 4 or 5 in $4 \%$. One hundred forty-nine (21\%) patients received extracorporeal life support device first for cardiogenic shock or bridge-to-candidacy, and then underwent switch to implantable devices (bridge-to-bridge). Overall survival rate after LVAD implantation was $90 \%$ at 1 year and $86 \%$ at 2 years. Competing outcome analyses demonstrated that only $10 \%$ of patients reached heart transplantation and 60\% were still on device therapy at 3 years. Causes of death were cerebrovascular accident in 44\%, infection in $18 \%$, bleeding in $6 \%$, and device failure in $6 \%$. Sixty-six per cent of patients required at least 1 hospital admission during an initial 1 year after device implantation.

In conclusion, continuous flow LVAD was effective for bridge-to-transplant. Although the collected data indicate that the improved survival rate is acceptable, there remains potential for major complications which precludes wider application of LVAD.

\section{DEVELOPING COMPREHENSIVE MECHANICAL CIRCULATORY SUPPORT IN SINGAPORE}

Cumaraswamy Sivathasan. Mechanical Circulatory Support and Transplantation, National Heart Centre Singapore, Singapore

10.1136/heartasia-2019-apahff.20

Heart transplantation was initiated in Singapore in 1990. Three to six heart transplants are performed annually. The waiting list mortality was about $30 \%$. Hence, mechanical circulatory support was initiated with HeartMate I in 2001. Extracorporeal membrane oxygenation (ECMO) for acute life support became available by 2003. Durable implantable rotary left ventricular assist device (LVAD) with HeartMate II $^{\mathrm{TM}}$ (HMII) was introduced in 2007, followed by HeartWare ${ }^{\mathrm{TM}}$ $\mathrm{HVAD}^{\mathrm{TM}}$ in 2012 and HeartMate $3^{\mathrm{TM}}$ (HM3) in 2015.

90 consecutive durable implantable devices (58 HMII, 21 HVAD and 11 HM3), were placed from May 2009 to December 2017. Of these, 65 were placed as bridge-to-transplant. Overall perioperative mortality was 7 (7.7\%). 26 were INTERMACS level 1 patients, bridged with ECMO without perioperative mortality. Median ICU stay and hospital stay were 8 and 31 days, respectively. Mean duration of support was 907 days. The longest support was 8.6 years. 45 had been on ongoing support, and 23 were transplanted. Late mortality occurred in 13 patients, 5 due to stroke. Driveline infection developed in 28.7\%. 57.1\% were associated with driveline trauma. Pocket infection developed in 5 patients. In the initial 78 patients, $17(21.7 \%)$ experienced gastrointestinal bleeding (GIB); 11 with recurrent GIB were successfully treated with thalidomide. 6 developed aortic regurgitation, 5 underwent modified Park stitch. Implantable cardioverter defibrillator placement pre-LVAD did not confer mortality benefit. Issues with hardware occurred in 60\%. Cardiac recovery occurred in 2 and their LVADs were decommissioned. Overall survival at 5 years was $84.4 \%$, compared with INTERMACS registry data of $48 \%$.

\section{SURGICAL CARE AND PERIOPERATIVE NURSING MANAGEMENT OF LVADS}

Jesus M Casida. Johns Hopkins School of Nursing, Baltimore, Maryland, USA

10.1136/heartasia-2019-apahff.21

The perioperative management of patients with a left ventricular assist device (LVAD) presents a whole set of challenges to the nurses at the bedside and outpatient settings. ${ }^{1}$ Despite the remarkable advancements in technology, the care of patients following LVAD implant remains complex for the multidisciplinary healthcare team. This presentation provides a brief overview of the currently used durable LVAD designs worldwide. These include axial (HeartMate $\mathrm{II}^{\mathrm{TM}}$ ) and magnetic flow levitation (HeartMate $3^{\mathrm{TM}}$ and HeartWare HVAD $^{\circledR}$ ) LVADs. $^{2}$ Discussion of the nursing management of patients post-LVAD implant in the critical care, progressive care, and in the outpatient care settings will be approached at conceptual level. This approach will equip the participant with a 'thinking framework' guiding his/her actions in caring for LVAD patients. Salient nursing actions include early detection and management of post-surgical complications, device-related complications, heart failure symptom exacerbations, infection, among others. The nurse's role in helping patients attain an optimum level of functioning post-implantation during hospitalisation and the impact of the nurse in supporting patients (and caregivers) to attain an increase in quality of life are highlighted.

\section{REFERENCES}

1. Chmielinski $A$, Koons $B$. Nursing care for the patient with a left ventricular assist device. Nursing 2018. 2017:47:34-40.

2. Montalto A, Loforte A, Musumeci F, Krabatsch T, Slaughter M (Eds.). Mechanical circulatory support in end-stage heart failure: a practical manual. Cham, Switzerland: Springer International Publishing; 2017. doi:10.1007/978-3-31943383-7

\section{RENAL COMPLICATIONS IN LVAD AND HEART TRANSPLANT PATIENTS}

Michael S Kiernan. CardiVascular Center, Tufts Medical Center and Tufts University School of Medicine, Boston, Massachusetts, USA

10.1136/heartasia-2019-apahff.22

Renal dysfunction remains a primary determinant of both short- and long-term outcomes following heart transplantation (HTx) and ventricular assist device (VAD) implantation. ${ }^{1}{ }^{2}$ Challenging to the assessment of candidacy for advanced heart failure (HF) therapies is the ability to distinguish intrinsic parenchymal renal disease from reversible cardiorenal disease. Patients with haemodynamically mediated renal failure may recover kidney function once renal perfusion is restored following HTx or VAD surgery. Chronic hypoperfusion, however, can lead to progressive structural lesions that may not improve despite correction of underlying haemodynamic abnormalities. In patients undergoing kidney biopsy as part of their HTx evaluation, there was poor correlation between eGFR and the extent of tubular atrophy and interstitial fibrosis. ${ }^{3}$ Prolonged elevation of serum creatinine (SCr) levels suggest but do not prove irreversibility and $\mathrm{SCr}$ in isolation is unlikely the best biomarker to determine transplant candidacy in this clinical setting. Reasons for SCr elevation in $\mathrm{HF}$ including pharmacotherapies such as renin-angiotensin- 